\title{
Editorial
}

\section{Neutrophil Rheology and Transit through Capillaries and Sinusoids}

In normal lungs, the concentration of neutrophils within the pulmonary capillary blood is about 40-80 times higher than it is within the blood in large vessels (1). This increased concentration, often referred to as the marginated pool, is thought to play an important role in host defense by allowing neutrophils time to sniff the local environment, looking for evidence of organisms or other stimuli that have escaped the initial barriers of host defense. The increased concentration is due to the difference in the pulmonary capillary transit times of neutrophils compared with erythrocytes (RBCS). Whereas R BC and plasma transit times measure 1-2 $\mathrm{s}$, Lien and Wagner have shown that the median transit time of neutrophils is $26 \mathrm{~s}$, with a wide range of 2 to more than $1,200 \mathrm{~s}(2,3)$. N eutrophils are not simply moving more slowly than R BCs; rather, they travel in hops through the highly anastomosing network of pulmonary capillary segments, with pauses followed by rapid travel. These observations suggest that a neutrophil moves rapidly through the larger capillary segments, but when it encounters a narrow one, it stops and requires time to deform. While neutrophils appear to elongate quickly when only small changes in shape are needed to enter a segment, deformation for entry into segments with diameters less than about $5.3 \mu \mathrm{m}$ requires a delay (4). This deformation time likely accounts for the longer transit times of neutrophils than R BCs and for the formation of the marginated pool. The large contribution of the deformation time to the total transit time points out the importance of the biomechanical properties of neutrophils, particularly their ability to deform, in the flow of these cells through the pulmonary capillary bed on the path from an arteriole to a venule.

The biomechanical properties of neutrophils are important not only in transit of neutrophils through healthy lungs but also during the inflammatory response in the capillaries of many organs including the lungs and liver (5-7). I nflammatory mediators, including IL-8, C 5a, PA F, LTB 4, FM LP, and TNF- $\alpha$, cause neutrophils to stiffen and become less deformable, most likely owing to the observed increase in $\mathrm{F}$-actin content within the submembrane region. These same mediators when infused intravascularly cause neutropenia owing to sequestration of neutrophils within the capillaries of the lungs and other organs. These observations have led to the hypothesis that stimulus-induced changes in the biomechanical properties of neutrophils, particularly a decreased ability to deform, traps neutrophils in capillaries after exposure to a stimulus within the blood stream or at sites of inflammation within the lung parenchyma when stimuli are present locally. Although a causal relationship has yet to be proved owing primarily to a lack of reagents that can specifically prevent this stiffening in vivo, considerable data are accumulating that support this hypothesis. For example, intravascular complement fragments

Supported by HL 48160, HL 52466, and HL 33009, and by a Clinical Scientist Award in Translational Research from the Burroughs Wellcome Fund.

Am J Respir Crit Care Med Vol 159. pp 1693-1695, 1999

Internet address: www.atsjournals.org cause neutrophil sequestration within the pulmonary capillaries, and the sequestered neutrophils are more round (less deformed), with a higher submembrane concentration of actin, than are neutrophils in the lungs of animals given control infusions (8). In contrast to the capillary beds of many organs, where the postcapillary venules are the site of neutrophil emigration, scientists dating back to Loosli and Baker in 1962 have shown that neutrophils sequester and emigrate through the pulmonary capillaries during the inflammatory response within the distal lung parenchyma (9). Selectin-mediated rolling of neutrophils along the endothelium, which is a critical first step in the adhesion cascade within the postcapillary venules and acts to slow neutrophils, does not occur in the pulmonary capillaries, presumably owing to spatial constraints (10). In fact, the selectins are often not required during the acute response of neutrophils, even for their signaling functions (11, 12). In the liver, sequestration of neutrophils within sinusoids is similarly independent of selectins (7). The stimulus-induced decrease in deformability likely replaces the role of selectinmediated rolling in stopping neutrophils within capillaries and sinusoids.

In this context, the studies of D rost and colleagues reported in this issue of The A merican Journal of Respiratory and Critical Care Medicine (pp. 1696-1702) make an important step forward in extending the observations made in the laboratory to understanding the rheologic properties of neutrophils in patients with sepsis. They used a cell transit analyzer, which measures the time required for a neutrophil to pass through a pore measuring $8 \mu \mathrm{m}$ in diameter and $20 \mu \mathrm{m}$ in length, to examine the pore transit time of neutrophils isolated from patients with sepsis. They convincingly show that the transit time through the pores is longer for neutrophils from patients with sepsis than from healthy subjects, and that circulating neutrophils from patients who improve clinically have pore transit times similar to those of normal subjects. Their data also demonstrate that treatment of neutrophils from healthy subjects with TNF- $\alpha$ results in increased pore transit times, similar to that observed in neutrophils from septic patients. Their work clearly and elegantly demonstrates that neutrophils circulating in septic patients are altered in their fundamental properties.

A s all scientists know, excellent studies raise more questions than they answer, and provoke many new thoughts and ideas. The work of $D$ rost and colleagues begs questions about the diameters of unstimulated neutrophils and the comparison between the diameters of neutrophils and the capillaries through which they must travel. R emarkably, the diameter of neutrophils is not yet known with the level of precision that is needed for understanding and modeling neutrophil trafficking. R eported values of neutrophil diameters vary from 6.8 to $8.3 \mu \mathrm{m}$. The techniques used to calculate this diameter include electron microscopy, Coulter counters, and micropipette aspiration, and each of these approaches has confounding artifacts. $V$ ariables include whether the microvillus processes on quiescent neutrophil surfaces are included in the diameter measurements. A lthough this reported range seems narrow, 
the range of capillary diameters is similar. Whether the majority of neutrophils are at the high or the low end of the reported range dramatically affects our estimates of both the number of capillaries too narrow for neutrophils to traverse without changes in their shape and, importantly, the deformation times required for the necessary shape changes.

The documentation by Drost and colleagues of a fundamental change in the ability of neutrophils to travel through $8.0 \mu \mathrm{m}$ pores also invites questions about the exact nature of this change and the mechanisms underlying it. They have carefully described the measurements made by the cell transit analyzer and its strengths and weaknesses. They suggest that one change in neutrophil behavior that could explain the lengthened pore transit time is a cytokine- or chemokine-induced increase in the rigidity of neutrophils, altering their ability to deform. A Ithough this change has likely occurred in neutrophils from septic patients, it is uncertain whether a decrease in deformability alone can account for the observed two- to threefold increase in transit times through $8.0 \mu \mathrm{m}$ pores, which actually require little deformation on the part of the neutrophils, even if neutrophil diameters in vivo are at the high end of the reported range. In addition, the changes in the cytoskeleton induced by inflammatory stimuli in vitro result in only a shortlived change in neutrophil deformability of 20-30 min (13), whereas the increase in pore transit time is observed several hours after the blood samples were obtained from patients. Perhaps any changes in the cytoskeleton that may be occurring in these patients arise through mechanisms different from those occurring after acute stimulation with inflammatory mediators. The changes in neutrophils from septic patients might involve more extensive remodeling of the cytoskeleton and the shape of the cells. Studies of neutrophil shape and of Factin content and distribution within the cytosol, as well as of the activities of signaling molecules thought to regulate $\mathrm{F}$-actin assembly, are exciting to contemplate.

A second possible explanation for the observed increase in pore transit time involves an increase in neutrophil volume in septic patients. G rinstein, $\mathrm{H}$ enson, W orthen, and other investigators have shown that activated neutrophils increase their volume by $20-100 \%$ through $\mathrm{Na}^{+} / \mathrm{H}^{+}$antiport activity (14), and this increase in volume could increase pore transit times. A third possibility concerns the binding of platelets to neutrophils in the blood through P-selectin and PSG L-1 interactions, which would increase the apparent volume of the neutrophils, prolonging their pore transit time. Fourth, increased adhesive interactions could be playing a role through the formation of neutrophil-neutrophil doublets. These doublets could enter the pores and therefore be measured. Whether the distribution of pore transit times became bimodal is not addressed by the authors. Finally, Hogg and colleagues, Lichtman and W eed, and members of our laboratory have demonstrated that neutrophils within and newly released from the bone marrow are less deformable and probably larger than neutrophils that have been circulating $(15,16)$. Sepsis stimulates release of neutrophils from the bone marrow, and the increase in rigidity observed by D rost and colleagues may reflect a higher fraction of newly released neutrophils in septic patients than in healthy subjects, rather than solely a stimulated or primed state. Studies pursuing these possibilities in more detail and their underlying mechanisms are exciting next steps that are likely to prove fruitful in understanding the rheology of neutrophils in these patients, as well as developing innovative approaches to therapy.

The neutrophils examined in this study were by necessity isolated from the blood and hence capable of circulating. $\mathrm{H}$ ow they compare with those that are sequestered in capillaries re- mains to be determined. Examining the frequency distribution of pore transit times for each patient to determine whether the increase in mean transit times reflects a lengthening for all neutrophils or very long transit times for a small population of circulating neutrophils will help to clarify the relationship between circulating and sequestered neutrophils. Whether stiffened neutrophils have longer transit times in vivo and whether longer transit times have physiological consequences for tissue injury are important but difficult questions to answer.

Drost and colleagues also examined the response of neutrophils from septic patients to subsequent inflammatory stimuli. They demonstrate that these neutrophils have an exaggerated response to $f M L P$, suggesting that their response to mediators encountered in the blood or tissues is enhanced compared with that of neutrophils from healthy subjects. These observations document in patients previous observations made in animals and in vitro, and underscore the need to understand the mechanisms. Whereas the signaling pathways underlying the effect of adhesion on subsequent responses of neutrophils and other cells are becoming clearer, the mechanisms mediating the observed effects in nonadherent neutrophils are poorly characterized and clearly important.

A Ithough the properties underlying the observed delay in pore transit time of neutrophils from septic patients are not yet clear, and the complexities of interpreting data obtained from virtually all excellent clinical studies are well recognized, the authors' observations are an important step forward in our understanding of neutrophil rheology during sepsis and other states in which circulating inflammatory mediators are present. The observed change in pore transit times may reflect altered trafficking of neutrophils in capillaries or sinusoids of all organs owing to lengthened capillary transit times. The defect in neutrophil transit may be accentuated in these hypotensive septic patients by a decrease in the pressure gradient from the proximal to the distal end of a capillary segment, which is thought to be the driving force that moves neutrophils through the capillaries. Whether the defects in pore transit times are sufficient to lengthen microvascular transit times in vivo, whether this increase in transit time enhances neutrophil-induced injury to endothelial cells in these patients, and whether the increase in neutrophil concentration resulting from any increase in transit time alters microvascular resistance and blood flow in septic patients are critical questions that remain to be addressed. The contribution these authors have made, their enthusiasm in pursuing in these difficult clinical studies, and their interests in extending the results of in vitro and in vivo studies in animals to the pathogenesis of disease in humans are to be applauded. They have confirmed and extended important concepts of neutrophil function, and their studies exemplify the need for clinical research in evaluating knowledge from laboratory studies within a clinical context and in bringing back well-focused new questions to the laboratory.

\author{
Claire M. Doerschuk \\ Physiology Program \\ D epartment of E nvironmental H ealth \\ $\mathrm{H}$ arvard School of Public $\mathrm{H}$ ealth \\ B oston, M assachusetts
}

\section{References}

1. Hogg, J. C., and C. M. D oerschuk. 1995. Leukocyte traffic through the lung. A nnu. Rev. Physiol. 57:97-114.

2. Hogg, J. C., T. M CL ean, B. A . M artin, and B. Wiggs. 1988. E rythrocyte transit and neutrophil concentration in the dog lung. J. A ppl. Physiol. 65:1217-1225. 
3. Lien, D. C., W. W. Wagner, J r., R . L. Capen, C. H aslett, W . L. H anson S. E . H ofmeister, P. M. H enson, and G . S. W orthen. 1987. Physiologic neutrophil sequestration in the canine pulmonary circulation. J. A ppl. Physiol. 62:1236-1243.

4. Wiggs, B. R ., D. English, W. M. Quinlan, N. A . D oyle, J . C. Hogg, and C. M. Doerschuk. 1994. The contributions of capillary pathway size and neutrophil deformability to neutrophil transit through rabbit lungs. J. A ppl. Physiol. 77:463-470.

5. Worthen, G. S., B. Schwab, E. L. Elson, and G. P. D owney. 1989. M echanics of stimulated neutrophils: cell stiffening induces retention in capillaries. Science 245:183-185.

6. Inano, H., D. English, and C. M. D oerschuk. 1992. E ffect of zymosan-activated plasma on the deformability of rabbit polymorphonuclear leukocytes and the role of the cytoskeleton. J. A ppl. Physiol. 73:13701376.

7. Jaeschke, H., and C. W. Smith. 1997. Cell adhesion and migration: III. L eukocyte adhesion and transmigration in the liver vasculature. A m. J. Physiol. 273 (G astrointest. L iver P hysiol. 36):G 1169-G 1173.

8. M otosugi, H ., L. G raham, T. W . N oblitt, N . A . D oyle, W . M . Q uinlan, Y . Li, and C. M. D oerschuk. 1996. Changes in neutrophil actin and shape during sequestration induced by complement fragments in rabbits. A m. J. Pathol. 149:963-973.

9. Loosli, C. G., and R. F. B aker. 1962. A cute experimental pneumococcal (type I) pneumonia in the mouse: the migration of leucocytes from the pulmonary capillaries into the alveolar spaces as revealed by the electron microscope. Trans. A m. Clin. Climatol. A ssoc. 74:15-28.
10. G ebb, S. A., J. A. Graham, C. C. H anger, P. S. Godbey, R. L. Capen, C. M. Doerschuk, and W. W. Wagner, J r. 1995. Sites of leukocyte sequestration in the pulmonary microcirculation. J. Appl. Physiol. 79:493-497.

11. M izgerd, J. P., B. B. M eek, G.J . K utkoski, D. C. B ullard, A . L. B eaudet, and C. M. D oerschuk. 1996. Selectins and neutrophil traffic: margination and Streptococcus pneumoniae-induced emigration in murine lungs. J. Exp. M ed. 184:639-645.

12. K ubo, H., N. A. D oyle, L. G raham, S. D. B hagwan, W. M. Q uinlan, and C. M. Doerschuk. 1999. L - and P-selectin and CD 11/CD 18 in intracapillary neutrophil sequestration in rabbit lungs. A m. J. Respir. Crit. Care M ed. 159:267-274.

13. Frank, R. S. 1990. Time-dependent alterations in the deformability of human neutrophils in response to chemotactic activation. Blood 76: 2606-2612.

14. G rinstein, S., W. Furuya, and E. J. Cragoe, J r. 1986. V olume changes in activated human neutrophils: the role of $\mathrm{Na}+/ \mathrm{H}+$ exchange. J. Cell. Physiol. 128:33-40.

15. V an E eden, F. J., Y . K itagawa, M. E . K lut, E . L awrence, and J . C. H ogg. 1997. Polymorphonuclear leukocytes released from the bone marrow preferentially sequester in the lung microvessels. M icrocirculation 4 369-380.

16. Lichtman, M . A ., and R . I. W eed. 1972. A Iternation of the cell periphery during granulocyte maturation: relationship to cell function. Blood 39:301-316. 